\title{
Development of Verruca Plana from Human Papillomavirus 78 Dependent on Host Immune State
}

Mayuna SHIMANO ${ }^{1}$, Toshinari MIYAUCHI ${ }^{1 *}$, Teruki YANAGI ${ }^{1}$, Toshifumi NOMURA ${ }^{1,2}$ and Hideyuki UJIIE ${ }^{1}$

${ }^{1}$ Department of Dermatology, Faculty of Medicine and Graduate School of Medicine, Hokkaido University, N15W7, Kita-Ku, Sapporo, 0608638 and ${ }^{2}$ Department of Dermatology, Faculty of Medicine, University of Tsukuba, Tsukuba, Japan. *E-mail: miyauchi@med.hokudai.ac.jp Accepted Nov 22, 2021; Epub ahead of print Nov 22, 2021

Verruca plana, also known as plane warts or flat warts, is a morphological form of benign wart caused by human papillomavirus (HPV) infection. More than 200 types of HPV have been identified, among which the Alphapapillomavirus 2 (aPV2) and, especially, HPV3 can cause verruca plana (1). Clinically, flat or slightly elevated papules are observed preferentially on the face and dorsal surfaces of the hands, especially in young people, but sometimes in elderly people, and the number of lesions ranges from a few to hundreds $(1-3)$. It is generally known that there are strong relationships between HPV types, clinical and histological morphologies, and anatomical sites or tissues, which can vary depending on the immune status of the host. We report here a case of verruca plana caused by HPV78 infection, which is rarely detected from immunocompetent individuals' lesions, in a patient with Sézary syndrome.

\section{CASE REPORT}

A 78-year-old Japanese man presented to our hospital with multiple flat papules on the extremities with un- known onset. He had been treated for erythroderma for 16 years with topical steroids, ultraviolet B phototherapy, and oral cyclosporine. He had been diagnosed with Sézary syndrome 4 years earlier, and had just undergone 4 months of mogamulizumab treatment. Physical examination revealed multiple light red, slightly itchy, flat papules of up to $3-5-\mathrm{mm}$ in diameter on the forearms and thighs (Fig. 1a). There were no lesions on the face or dorsal surfaces of the hands. In dermoscopic findings, red dots and globular vessels were observed. Histology of a flat papule on the right forearm showed hyperkeratosis and acanthosis, with hypergranulosis and numerous koilocytes in the upper epidermis. To determine the type of HPV in the lesions, HPV genotyping was performed by restriction fragment length polymorphism (RFLP). This is a modified version of a previously reported method (4), in which HPV genotypes can be distinguished, based on the cutting patterns of PCR products digested with some restriction enzymes (Fig. 1b). Total DNA was extracted from a lesion on the left thigh using the QIAamp DNA Mini Kit (Qiagen, Hilden, Germany) and amplified by PCR using AmpliTaq Gold 360 Master Mix (Applied
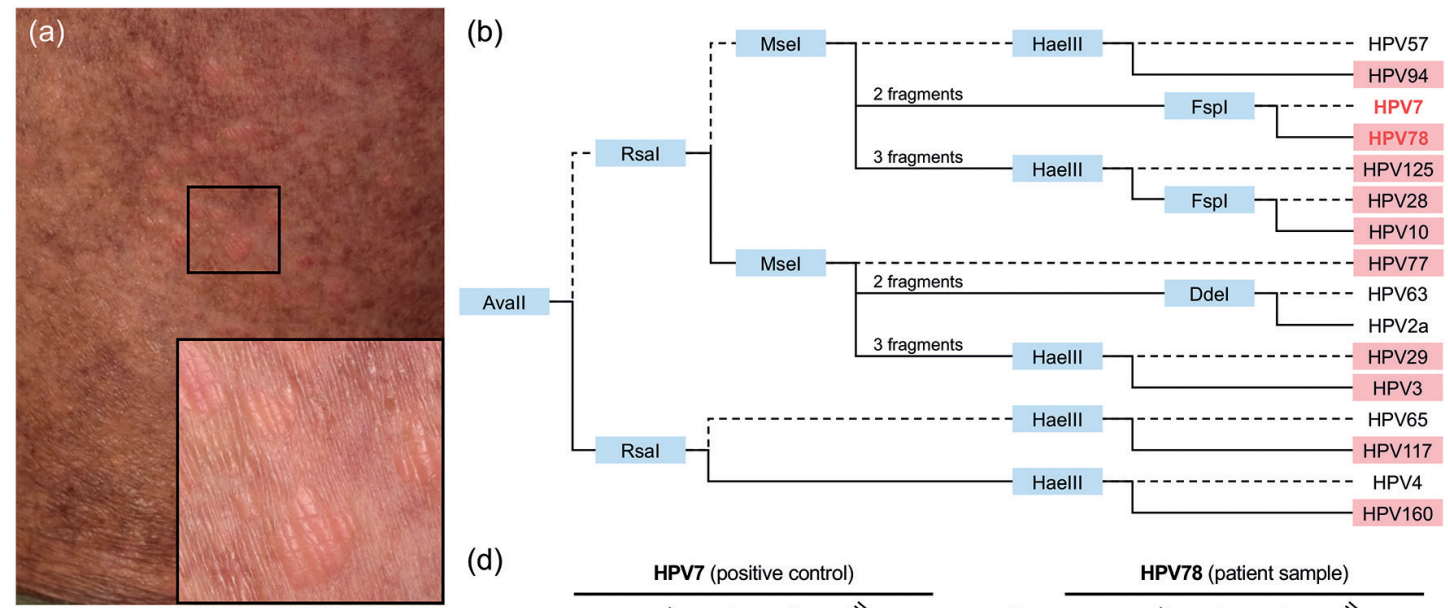

(d)
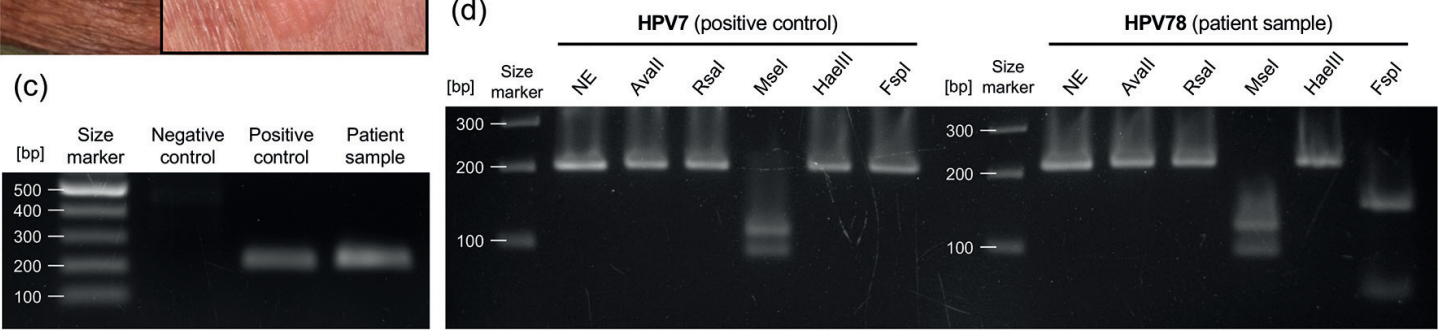

Fig. 1. Multiple flat papules on the right thigh. (a) Inset: high magnification of the area indicated in the box. (b) A flow chart of HPV-genotyping using restriction fragment length polymorphism (RFLP). HPV types including aPV2 (pink) can be distinguished through the cutting patterns by restriction enzymes (blue). Solid lines represent "cut" and dotted lines "not cut". (c) Electrophoretic profile of the PCR samples. DNA extracted from normal human skin was used as a negative control, and that from a HPV7-related wart as a positive control. The estimated size of the amplicon is approximately 220 bp. (d) Results of digestion with 5 restriction enzymes. NE: no enzyme. 
Biosystems, Foster City, CA) and a specific primer set for detection of aPV2 (4) (Fig. 1c). The PCR amplification conditions were as follows: 1 cycle at $96^{\circ} \mathrm{C}$ for 10 min; 35 cycles at $96^{\circ} \mathrm{C}$ for $30 \mathrm{~s}, 45^{\circ} \mathrm{C}$ for $1 \mathrm{~min}$ and $72^{\circ} \mathrm{C}$ for $1 \mathrm{~min}$; then 1 cycle at $72^{\circ} \mathrm{C}$ for $7 \mathrm{~min}$. PCR products were then digested with 5 restriction enzymes (AvaII, RsaI, MseI, HaeIII, FspI) according to the manufacturer's instructions. This procedure revealed HPV78 DNA in the patient sample (Fig. 1d), which could be confirmed by subsequent sequencing after TA cloning. Furthermore, 4 different multiplex primer sets for the detection of other types of HPV (5) failed to detect any other type in the lesions. Based on the above information, we finally diagnosed the patient with verruca plana caused by HPV78 infection, and treated with cryotherapy.

\section{DISCUSSION}

HPV78 was first identified and registered with the Karolinska Institute's HPV reference centre in 1995 (details unavailable). In 2000, 3 cases in which HPVXS2 (later discovered to be HPV78) was detected from squamous cell carcinoma and actinic keratosis of renal transplant recipients were reported (6). Since then, 7 additional cases of HPV78-related skin lesions have been reported (7-9). Among these 10 cases, 8 were associated with immunosuppressed or immunocompromised states due to renal transplantation (4/10), HIV infection (3/10), or splenectomy (1/10). For the remaining 2 cases, HPV78 was detected in skin samples taken from immunocompetent individuals by transcriptome analysis. Interestingly, 1 of the samples that demonstrated HPV78 was normal control skin, while the other was a wart sample. From these data, it can be inferred that, while HPV78 may be present in normal skin, whether it forms skin lesions depends on the immune state of the host. Mitsuishi et al., in their HPV screening of flat warts taken from immunocompetent patients, did not detect HPV78 (1), further supporting the theory that host immune condition is an important factor. In the current case, both Sézary syndrome itself and the various types of longstanding immunosuppressive therapies for erythroderma the patient had undergone would presumably have affected his immune state and caused HPV78-related warts to develop.

Although it is generally recognized that the lesions of verruca plana commonly appear on the face and dorsum of the hands, in the current case they were atypically and widely distributed only on the extremities and not on the face. Conversely, the dermoscopic findings of each lesion were quite similar to those reported previously (10); hence the characteristics of each lesion were typical. Regression of the lesions can occur in verruca plana, which is one of its main clinical features. However, the current case had not followed this course, and cryotherapy had had a limited effect thus far. This could be characteristic of HPV78-associated verruca plana, which is presumably the result of infection against a background of host immune status.

These findings suggest that whether HPV78 develops skin lesions depends largely on the host's immune state. However, the amount of data currently available on HPV78 is limited, and further study is necessary.

The authors have no conflicts of interest to declare.

\section{REFERENCES}

1. Mitsuishi T, Ohsawa I, Kato T, Egawa N, Kiyono T. Molecular cloning and characterisation of a novel type of human papillomavirus 160 isolated from a flat wart of an immunocompetent patient. PLoS One 2013; 8: 2-6.

2. Kyriakis KP, Pagana G, Michailides C, Emmanuelides S, Palamaras I, Terzoudi S. Lifetime prevalence fluctuations of common and plane viral warts. J Eur Acad Dermatol Venereol 2007; 21: 260-262.

3. Masatkar V, Gupta LK, Khare AK, Mehta S, Mittal A. Clinicoepidemiologic study of verruca plana at a tertiary care center. Indian J Dermatol Venereol Leprol 2018; 84: 247.

4. Sasagawa T, Mitsuishi T. Novel polymerase chain reaction method for detecting cutaneous human papillomavirus DNA. J Med Virol 2012; 84: 138-144.

5. Miyauchi T, Moriuchi R, Hamade Y, Suzuki S, Nomura T, Shimizu S. Warts in toe webs associated with human papillomavirus type 7: a specific cutaneous manifestation of this type? $\mathrm{Br}$ J Dermatol 2016; 174: 678-681.

6. Berkhout RJM, Bouwes Bavinck JN, ter Schegget J. Persistence of human papillomavirus DNA in benign and (pre) malignant skin lesions from renal transplant recipients. J Clin Microbiol 2000; 38: 2087-2096.

7. Silling S, Wieland U, Werner M, Pfister $H$, Potthoff A, Kreuter A. Resolution of novel human papillomavirus-induced warts after HPV vaccination. Emerg Infect Dis 2014; 20: 142-145.

8. Devitt K, Hanson SJ, Tuong ZK, McMeniman E, Soyer HP, Frazer IH, et al. Single-cell RNA sequencing reveals cell type-specific HPV expression in hyperplastic skin lesions. Virology 2019; 537: 14-19.

9. Al-Eitan LN, Tarkhan AH, Alghamdi MA, Al-Qarquz FA, AlKofani HS. Transcriptome analysis of HPV-induced warts and healthy skin in humans. BMC Med Genomics 2020; 13: 1-10.

10. Kim WJ, Lee WK, Song M, Kim HS, Ko HC, Kim BS, et al. Clinical clues for differential diagnosis between verruca plana and verruca plana-like seborrheic keratosis. J Dermatol $2015 ; 42$ : 373-377. 